\title{
A NEW METHOD FOR THE QUANTITATIVE SEPARATION OF STRYCHNINE AND QUININE.
}

G. N. WATSON, LNIVERSity OF KANSAS.

The chloroplatinate of strychnine is nearly insoluble in water, insoluble in alcohol and practically insoluble in a mixture of alcohol, $90 \%$ and dilute hydrochloric acid, $10 \%$. The chloroplatinate of quinine is soluble in water, soluble in alcohol and very readily soluble in the alcohol-hydrochloric acid mixture.

From the above clata, the following method has been worked out for the separation of strychnine and quinine that seems to excel in point of accuracy and simplicity any method heretofore published,- the method being especially valuable for the separation of small quantities of strychnine from large quantities of quinine, a condition generally found when these two drugs are prescribed together.

Dissolve .050 to .100 grammes of the mixed alkaloids (depending on amount of strychnine present) in a small amount of the alcohol-hydrochloric acid mixture (about $5 \mathrm{cc}$.), add $20 \%$ solution of platinic chloride, drop by drop, while slightly agitating the mixture, until the precipitation is complete. Add $5 \mathrm{cc}$. more of the solvent, cover with watch glass and set aside for one hour, then filter through tared filter, wash with alcohol, place in oven, dry at $100^{\circ} \mathrm{C}$. for fifteen minutes, cool and weigh. If the proportion of quinine is large, it will be necessary to add $5-15 \mathrm{cc}$. more of the solvent before filtering. It will also be necessary to decompose the precipitate with alkali, $\mathrm{NaOH}$, recover the strychnine with chloroform, evaporate and reprecipitate the chloroformic residue from a few cc. of the alcohol-hydrochloric acid solvent, with the platinic chloride reagent.

The chloroplatinate of strychnine is yellow in color, crystalline, and has a remarkable lustre. It contains about $62 \%$ of the alkaloid and $18 \%$ of metallic platinum.

The chloroplatinate of quinine has an orange color and an amorphous appearance.

A trace of chloroplatinate of quinine gives an amorphous appearance to the chloroplatinate of strychnine, a valuable indication of the purity of the strychnine salt.

The amount of strychnine can be calculated from the weight of the chloroplatinate, from the amount of metallic platinum, or directly by the decomposition of the chloroplatinate.

By this method it has been found possible to determine the strychnine in a mixture of .060 of quinine and .002 of strychnine, being about the proportions found in such preparations as Elixir of Iron. Quinine and Strychnine.

In determining the strychnine in the Elixir of Iron, Quinine and Strychnine, it would be necessary to liberate the total alkaloids and proceed with the mixed alkaloids as directed. The quinine can be determined by difference or from the filtrate by making alkaline, shaking out with ether or chloroform and weighing the dried residue.

Gold chloride produces with strychnine a salt having a solubility and other properties very similar, if not identical, with that of the platinic chloride. 